\title{
Scriptaid, a novel histone deacetylase inhibitor, enhances the response of human tumor cells to radiation
}

\author{
TAKESHI KURIBAYASHI, MAKI OHARA, SAKURA SORA and NOBUO KUBOTA \\ Department of Radiological Sciences, Ibaraki Prefectural University of Health Sciences, \\ 4669-2 Ami, Ami-machi, Inashiki-gun, Ibaraki 300-0394, Japan
}

Received July 7, 2009; Accepted August 17, 2009

DOI: 10.3892/ijmm_00000309

\begin{abstract}
A group of histone deacetylase (HDAC) inhibitors has been shown to suppress the growth of a variety of human tumor lines in vitro and in vivo and they are among the most promising candidates for anti-cancer therapeutic agents. We investigated the ability of scriptaid, a novel HDAC inhibitor and trichostatin A (TSA) to enhance cell killing by radiation in radioresistant SQ-20B cells derived from human head and neck squamous carcinoma. SQ-20B cells were treated with scriptaid or TSA in combination with radiation. Cell survival was determined by a colony formation assay and protein levels were examined by Western blotting. DNA double strand breaks were measured by a $\gamma$-H2AX focus assay. Radiosensitization was observed for SQ-20B cells incubated with scriptaid at $5 \mu \mathrm{M}$ or TSA at $0.1 \mu \mathrm{M}$ for $24 \mathrm{~h}$. Radiosensitization by scriptaid was accompanied by a prolonged retention of $\gamma$-H2AX foci, suggesting that the enhancement of radiation cell killing by scriptaid involved inhibition of DNA double strand break repair. In addition, treatment with scriptaid suppressed expression of Ku80, but not Ku70. Scriptaid may be a useful radiosensitizer in the treatment of radioresistant human carcinomas.
\end{abstract}

\section{Introduction}

Chromatin remodeling plays an important role in transcriptional regulation. In eukaryotic cells, DNA is packaged into chromatin and dynamic processes are required to alter gene expression. Acetylation/deacetylation of histones is one such mechanism facilitated by histone acetyltransferases and histone deacetylases (HDAC), which are emerging as important molecules in transcriptional regulation (1). Acetylation of lysine residues in histones results in more open chromatin structure and activation of transcription. Hypoacetylation of histones results in condensed chromatin structure and

Correspondence to: Dr Nobuo Kubota, Department of Radiological Sciences, Ibaraki Prefectural University of Health Sciences, 4669-2 Ami, Ami-machi, Inashiki-gun, Ibaraki 300-0394, Japan

E-mail:kubota@ipu.ac.jp

Key words: radiosensitization, scriptaid, $\gamma-\mathrm{H} 2 \mathrm{AX}$, histone deacetylase inhibitor, Ku80 repression of gene transcription. Aberrant regulation of histone acetylation is among the epigenetic modifications that contribute to inappropriate gene expression in cancer cells. HDACs participate in this mode of gene regulation and are recognized as promising targets for cancer therapy $(2,3)$. Trichostatin A (TSA) has been used in studies investigating the role of HDAC inhibition on gene expression. However, TSA is of limited therapeutic use due to poor stability in vivo and high toxicity. Structurally varied HDAC inhibitors have been discovered, including hydroximates, cyclic peptides, aliphatic acids and benzamides (4). HDAC inhibitors induce differentiation, growth arrest and apoptosis in tumor cells in vitro and inhibit tumor growth in animals in vivo (5-9). Several such drugs are now in clinical trials.

The HDAC inhibitors have shown synergistic anti-tumor effects with a wide range of chemotherapeutic agents (10). In addition, several studies have shown that HDAC inhibitors, including TSA (11), MS-275 (12), sodium butyrate $(13,14)$, suberoylanilide hydroxamic acid (SAHA) (15) and PCI-24781 (16), sensitize human tumor cells to ionizing radiation.

Using a high-throughput system based on a stably integrated transcriptional reporter to screen a library of compounds, a novel HDAC inhibitor, scriptaid, was identified. Scriptaid belongs to an existing class of hydroxamic acid-containing HDAC inhibitors and is less toxic than TSA (17). It has been shown previously that scriptaid and TSA induce growth inhibition, cell cycle arrest and apoptosis in human cells, suggesting recovery of tumor suppressor function in response to histone deacetylase $(18,19)$. These data indicate that scriptaid is a promising candidate for further study in cancer therapy. In this study, we investigated the effect of scriptaid on the radiosensitivity of human tumor cells. The data presented indicate that scriptaid enhances the response of human tumor cells to radiation and the radiosensitizing effect is associated with a decreased capacity of cells to repair DNA double strand breaks.

\section{Materials and methods}

Cell culture and chemicals. The human squamous cell carcinoma cell line SQ-20B was maintained in $\alpha$-minimal essential medium ( $\alpha$-MEM) supplemented with $20 \mathrm{mM} 4-(2-$ hydroxyethyl) pipderazineethane sulphonic acid (HEPES), $8 \mathrm{mM} \mathrm{NaHCO}_{3}$, streptomycin, penicillin and $10 \%$ fetal bovine serum. Cells were cultured in a humidified incubator at $37^{\circ} \mathrm{C}$ 
with a mixture of $98 \%$ air and $2 \% \mathrm{CO}_{2}$. Scriptaid (6-(1,3dioxo- $1 H, 3 H$-0benzo[de]isoquinolin-2-yl)-hexanoic acid hydroxyamide) and TSA were purchased from Sigma (St. Louis, MO, USA).

Irradiation. Cells were irradiated with $10 \mathrm{MV}$ X-rays from a linear accelerator (Mitsubishi Medical Linac, Mitsubishi Electric, Tokyo, Japan) at a dose rate of $\sim 4 \mathrm{~Gy} / \mathrm{min}$. Doses were measured using an Innax Dosemaster (NE Technology, Berks, UK) before irradiation.

Clonogenic assay. Cell survival was measured by a colony formation assay (20). Briefly, cells were seeded in $24-\mathrm{cm}^{2}$ flasks and incubated for $\sim 24 \mathrm{~h}$ before treatment with scriptaid or TSA. Cells were exposed to scriptaid or TSA for $16 \mathrm{~h}$, irradiated with X-rays and incubated in the presence of the drug for a further $8 \mathrm{~h}$. Cells were washed with Dulbecco's phosphate-buffered saline (PBS) and dispersed with $0.05 \%$ trypsin containing $0.02 \%$ EDTA. Single cells were counted and seeded in $60-\mathrm{mm}$ dishes at various cell densities. The dishes were incubated in a $\mathrm{CO}_{2}$ incubator for 12 days. Colonies were stained with crystal violet dissolved in $20 \%$ methanol. Colonies of $>50$ cells were counted as survivors.

Assessment of apoptosis. Induction of apoptosis was assayed by detecting apoptotic bodies. Cells were exposed to scriptaid for $16 \mathrm{~h}$ and irradiated with X-rays (6 Gy). The cells were incubated in the presence of scriptaid for a further $8 \mathrm{~h}$. The medium was then removed and replaced with fresh medium free of the drug. Twenty-four hours after X-irradiation, both floating and attached cells were collected by centrifugation, resuspended in a fixative solution containing $3 \%$ paraformaldehyde in PBS and stained with the Hoechst 33258 fluorescent DNA stain (Sigma). The cells were placed on microscope slides and covered with glass cover slips. The number of apoptotic cells was counted and cells were photographed using a fluorescence microscope.

Western blot analysis. Cells were treated with scriptaid for $24 \mathrm{~h}$. In the case of a combination of scriptaid and Xirradiation, cells were treated with scriptaid for $16 \mathrm{~h}$, irradiated with X-rays and incubated in the presence of the drug for a further $8 \mathrm{~h}$. Cells were lysed in lysis buffer (Cell Signaling Technology, Beverly, MA, USA) and then frozen and thawed three times. The lysates were centrifuged at 15,000 rpm to remove any cellular debris. Protein levels were quantified using a BCA protein assay kit (Pierce, Rockford, IL, USA). Proteins were resolved by electrophoresis on $7.5 \%$ polyacrylamide gels and transferred onto polyvinylidene difluoride (PVDF) membranes (20). Target protein levels were assessed using antibodies to Bcl-2, Apaf-1, Ku70, Ku80 (Cell Signaling Technology) and actin (Chemicon International Inc., Temecula, CA, USA). Primary antibodies were detected using horseradish peroxidase (HRP)-conjugated secondary antibodies and the enhanced chemiluminescence (ECL) detection system (Amersham, Piscataway, NJ, USA).

Immunofluorescent staining for $\gamma-H 2 A X$. Cells were grown on glass slides placed in 60-mm dishes and exposed to scriptaid at $5 \mu \mathrm{M}$ for $16 \mathrm{~h}$, irradiated with $\mathrm{X}$-rays of $1 \mathrm{~Gy}$ and incubated
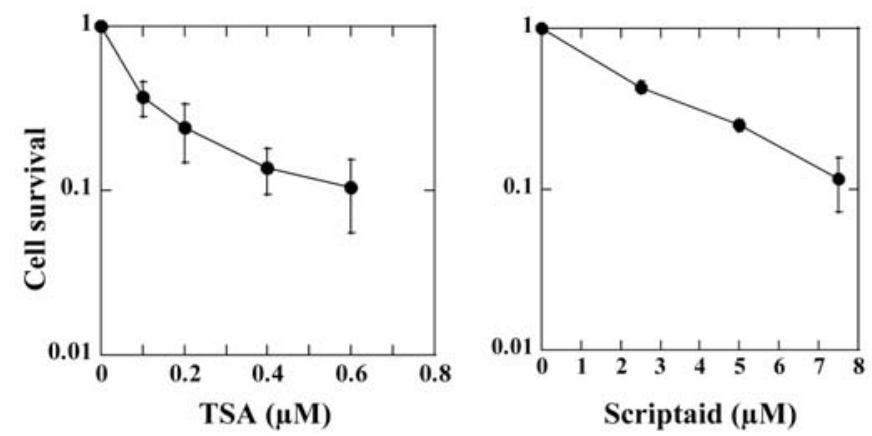

Figure 1. Effect of TSA or scriptaid on survival of SQ-20B cells. Cells were exposed to either drug for $24 \mathrm{~h}$ at $37^{\circ} \mathrm{C}$ and cell survival was measured by colony formation assay. Data points and bars represent the average and standard errors, respectively, from two or three separate experiments.

in the presence of the drug for a further $8 \mathrm{~h}$. At specified times, medium was removed, and cells were fixed with cold methanol for $20 \mathrm{~min}$ followed by acetone for 5-10 sec. Cells were blocked with $10 \%$ bovine serum albumin in PBS for $20 \mathrm{~min}$, washed twice with PBS and incubated with anti- $\gamma-\mathrm{H} 2 \mathrm{AX}$ antibody (Upstate Biotechnology, Charlottesville, VA, USA) for $1 \mathrm{~h}$. Cells were again washed twice with PBS before incubating in the dark with a FITC-labeled secondary antibody for $1 \mathrm{~h}$. Cells were washed twice with PBS, incubated in the dark with 4',6-diamidino-2-phenylindole (DAPI) in PBS for $5 \mathrm{~min}$ and again washed twice. Cover slips were mounted and slides were examined with a fluorescence microscope (Olympus, Japan). Images were captured by a CCD camera and $\gamma-\mathrm{H} 2 \mathrm{AX}$ foci were counted in at least 50 cells.

\section{Results}

Radiosensitization by scriptaid. First, we examined the effect of scriptaid and TSA on survival of SQ-20B cells by a colony formation assay. Cells were treated with various concentrations of the drugs for $24 \mathrm{~h}$. Dose-dependent curves of cell survival of SQ-20B cells treated with scriptaid or TSA are shown in Fig. 1. To assess the effects of scriptaid on the radiosensitivity of SQ-20B cells, we chose the concentration of $5 \mu \mathrm{M}$ of scriptaid and $0.1 \mu \mathrm{M}$ of TSA, respectively, which allowed cell survival at a rate of $0.3-0.4$. We constructed cell survival curves for SB-20B cells after X-irradiation in the presence or absence of scriptaid and TSA. The cells were exposed to scriptaid or TSA for $16 \mathrm{~h}$ at $37^{\circ} \mathrm{C}$, irradiated with X-rays and incubated for an additional $8 \mathrm{~h}$. SQ-20B cells showed a significant increase in radiation-induced cell death when treated with scriptaid or TSA (Fig. 2). The radiosensitivity enhancement ratios measured at a survival level of $10 \%$ were 1.4 and 1.5 , respectively.

Effect of scriptaid on apoptosis. Fig. 3a shows the percentage of apoptotic cells $24 \mathrm{~h}$ after X-irradiation. SQ-20B cells exhibit a relatively low basal level of apoptosis. After exposure of cells to X-rays with and without scriptaid, no significant increase in apoptosis was observed. The protein level of Bcl-2 remained essentially unchanged after drug treatment, $\mathrm{X}$-irradiation or both (Fig. 3b). In addition, the expression of Apaf-1, which regulates the activation of caspase-9, was 


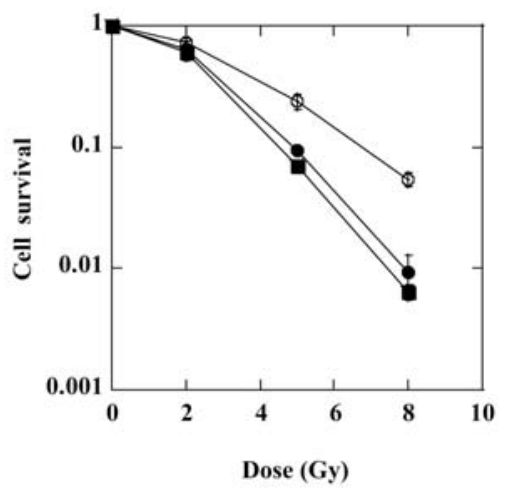

Figure 2. Radiosensitization of SQ-20B cells by scriptaid or TSA. Cells were incubated with DMSO $(\bigcirc), 5 \mu \mathrm{M}$ scriptaid $(\bullet)$ or $0.1 \mu \mathrm{M}$ TSA (घ) for $16 \mathrm{~h}$, irradiated with $\mathrm{X}$-rays and then incubated for a further $8 \mathrm{~h}$. Thereafter, cells were trypsinized, counted and plated in 60-mm dishes. Data points and bars represent the average and standard errors, respectively, from two or three separate experiments.

$\mathbf{a}$

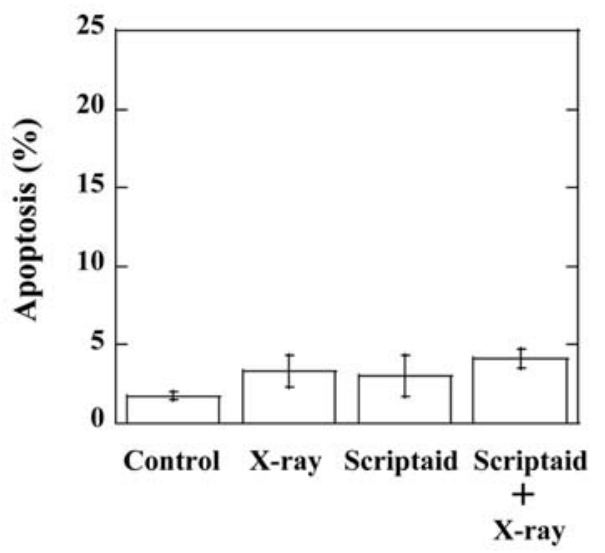

$\mathrm{b}$

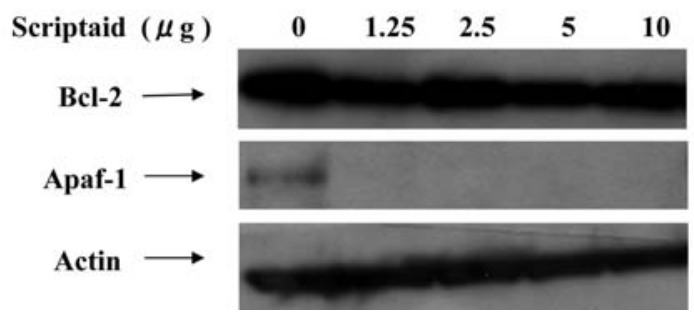

Figure 3. (a) Apoptosis in SQ-20B cells following X-irradiation (6 Gy), scriptaid treatment $(5 \mu \mathrm{M})$ or both. Apoptotic cells were measured $24 \mathrm{~h}$ after $\mathrm{X}$-irradiation. Error bars represent standard errors from three separate experiments. (b) Effect of scriptaid on the expression of apoptosis-related proteins in SQ-20B cells. Whole lysates were subjected to Western blotting for Bcl-2 and Apaf-1. Western blots for actin are shown as loading controls.

downregulated by scriptaid. These data suggest that SQ-20B cells are radiosensitized without a significant effect on apoptotic cell death.

Effect of scriptaid on the retention of radiation-induced $\gamma$ $H 2 A X$ foci and expression of DNA repair-related proteins. We measured the effect of scriptaid on the retention of radiationinduced $\gamma-\mathrm{H} 2 \mathrm{AX}$ foci, an indicator of DNA double strand breaks, in SQ-20B cells. The number of $\gamma-\mathrm{H} 2 \mathrm{AX}$ foci per cell was counted and the results indicate that more foci formed in cells irradiated with X-rays and incubated with scriptaid 4 to $24 \mathrm{~h}$ after irradiation than with X-rays alone (Fig. 4). To investigate the mechanism responsible for scriptaid-mediated prolongation of $\gamma-\mathrm{H} 2 \mathrm{AX}$ foci after irradiation, we examined the effect of scriptaid on the expression of DNA repair-related proteins $\mathrm{Ku} 70$ and $\mathrm{Ku} 80$. Cells were exposed to scriptaid at various concentrations for $24 \mathrm{~h}$ and then were collected and lysed for immunoblot analysis. As shown in Fig. 5, scriptaid downregulated expression of Ku80, but not Ku70, in SQ-20B cells. The decrease in Ku80 expression was evident at a $1.25 \mu \mathrm{M}$ concentration of scriptaid. In addition, there was a decrease in the level of Ku80 protein after scriptaid treatment in X-irradiated cells. These data suggest that Ku80 downregulation induced by scriptaid seems to play an important role in inhibiting the rejoining of radiation-induced DNA double strand breaks.

\section{Discussion}

It is known that HDAC inhibitors induce cell cycle arrest, terminal differentiation, mitotic cell death and inhibition of angiogenesis in many human tumor cell lines and in vivo tumors. In addition, they activate extrinsic and intrinsic apoptosis pathways $(4,21)$. In comparison, normal cells are more resistant to HDAC inhibitor-induced cell death (9). Thus, HDAC inhibitors that induce differentiation and/or death of tumor cells may provide an alternative or additional approach to the treatment of cancers.

Sun et al identified a novel HDAC inhibitor, scriptaid, using a high-throughput system based on a stably integrated transcriptional reporter to screen the entire library consisting of 16,320 compounds (17). Scriptaid belongs to an existing class of hydroxamic acid-containing HDAC inhibitors, which includes TSA. Scriptaid and TSA possess the same hydroxamic acid group, an aliphatic chain and an aromatic cap at the other end and both have a five-carbon link between a bulky end group and the hydroxamic acid moiety. Scriptaid has been reported to increase the levels of acetylated histones $\mathrm{H} 3$ and $\mathrm{H} 4$ in cancer cells and to be highly effective in suppressing the growth of human endometrial and ovarian cancer cells with little effect on normal human endometrial epithelial cells (19). These findings suggest that scriptaid may be a useful agent with relatively low toxicity for the treatment of carcinoma.

Radiotherapy is an important component in the treatment of cancers. However, radiotherapy alone often fails to suppress tumor growth in advanced stages. It is generally considered that the presence of inherently radioresistant tumor cells is one of the reasons for radiation therapy failure. Thus, radiosensitivity of tumor cells is a major determinant of local control (22). The HDAC inhibitors have shown synergistic cell killing for human tumor cell lines with chemotherapeutic drugs $(23,24)$. In addition, several HDAC inhibitors have been reported to enhance cell killing by ionizing radiation in human tumor cells $(8,12-14,16)$. In this study, we demonstrated that the HDAC inhibitor scriptaid also sensitized human tumor cells to radiation. It has been reported that HDAC inhibitors are potent inducers of apoptotic cell death (9). After exposure of cells to X-rays with and without scriptaid, no significant increase of apoptosis was observed, indicating the mechanisms underlying scriptaid- or TSA-induced radiosensitization of 
$\mathbf{a}$

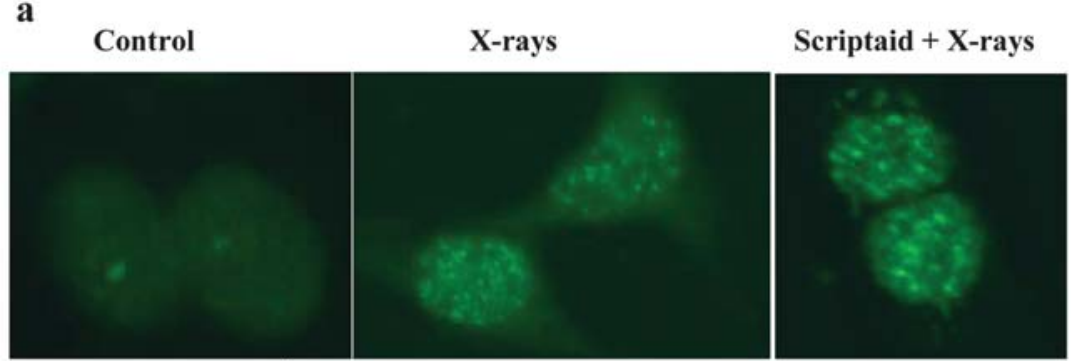

b

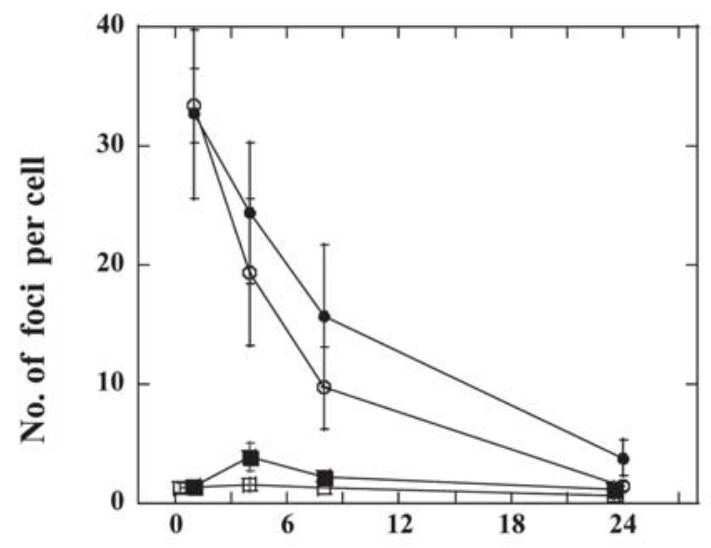

Time after $\mathrm{X}$-irradiation (h)

Figure 4. (a) Micrographs of $\gamma$-H2AX foci from cells taken $1 \mathrm{~h}$ after exposure to X-rays alone or X-rays in combination with scriptaid. (b) Quantitative analysis of $\gamma$-H2AX foci/cell following X-irradiation, incubation with $5 \mu \mathrm{M}$ scriptaid or both in SQ-20B cells. Cells were exposed to scriptaid for $16 \mathrm{~h}$, irradiated with X-rays (1 Gy) and incubated in the presence of the drug for a further $8 \mathrm{~h}$. Then cells were changed to fresh medium without the drug. Control ( $\square$ ), scriptaid alone ( $\square$ ), $\mathrm{X}$-ray alone $(\bigcirc)$, scriptaid $+\mathrm{X}$-ray $(\bullet)$. Error bars represent standard errors from two or three separate experiments.

a
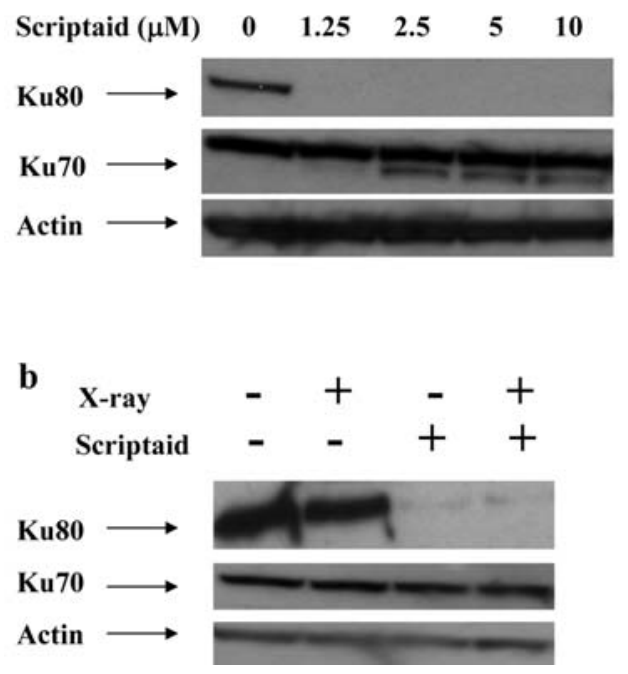

Figure 5. Effect of scriptaid on the expression of Ku70 and Ku80 in SQ-20B cells. (a) Cells were treated with scriptaid at various concentrations for $24 \mathrm{~h}$. (b) Cells were treated with scriptaid $(5 \mu \mathrm{M})$ for $16 \mathrm{~h}$, irradiated with X-rays and incubated in the presence of the drug for a further $8 \mathrm{~h}$. Whole cell lysates were subjected to Western blotting for Ku70 and Ku80. Western blots for actin are shown as loading controls.

SQ-20B cells do not involve an increase in apoptosis. Similar results were reported in TSA-mediated radiosensitization in SQ-20B cells (11).
Recently, $\gamma-\mathrm{H} 2 \mathrm{AX}$ expression has been established as a sensitive indicator of DNA double strand breaks induced by low doses of radiation. The histone $\mathrm{H} 2 \mathrm{AX}$ is phosphorylated $(\gamma-\mathrm{H} 2 \mathrm{AX})$ at sites of radiation-induced DNA double strand breaks and readily forms $\gamma-\mathrm{H} 2 \mathrm{AX}$ foci, which are visible by immunofluorescence staining (25). In several studies on HDAC-mediated radiosensitization in human tumor cells, the prolonged retention of radiation-induced $\gamma-\mathrm{H} 2 \mathrm{AX}$ foci, a hallmark of DNA double strand breaks, was observed in tumor cells after treatment with a combination of radiation and HDAC inhibitors when compared to radiation alone $(12,15)$. These findings indicate that the mechanism responsible for HDACmediated radiosensitization is the inhibition of DNA double strand breaks. Recently, it has been reported that sodium butyrate $(\mathrm{NaB})$ and SAHA (vorinostat) suppressed the levels of $\mathrm{Ku} 70, \mathrm{Ku} 80$ and $\mathrm{Rad} 50$ proteins $(14,15)$, which play a role in the non-homologous end-joining pathway for DNA double strand break repair (26). In the present study, the number of radiation-induced $\gamma$-H2AX foci was higher in cells exposed to X-rays in combination with scriptaid 4-24 h after Xirradiation than in cells exposed to X-rays alone. We also examined the effect of scriptaid on the levels of Ku70 and Ku80 in SQ-20B cells. Treatment with scriptaid suppressed the expression of Ku80, but not Ku70 (Fig. 5), suggesting that $\mathrm{Ku} 80$ is the target of radiosensitization by scriptaid in SQ-20B cells. Ku80 is an important component of the DNA double strand repair system (27) and cells deficient in the Ku80 protein are hypersensitive to radiation $(28,29)$. It is well known 
that oncogenic mutations in HRAS, which frequently occur in many types of cancers, could contribute to radiation resistance of tumor cells $(30,31)$. It has been shown that Ku 80 contributes to the oncogenic HRAS-mediated increase in the capacity of NIH3T3 cells to repair DNA double strand breaks and to afford protection against ionizing radiation (32). Taken together, it is considered that scriptaid sensitizes SQ-20B cells to radiation by reducing the expression of $\mathrm{Ku} 80$ and subsequently inhibiting DNA double strand break repair by a non-homologous end-joining pathway.

In summary, the present study indicates that the molecular mechanism underlying radiosensitization by scriptaid is due, at least in part, to the downregulation of the Ku80 protein. Our results show that scriptaid is a promising agent that may be useful in combination with radiotherapy against radioresistant carcinoma.

\section{Acknowledgements}

This study was supported in part by a Grant-in-Aid for Scientific Research from the Japan Society for the Promotion of Science (18591388) and by a Grant-in-Aid for Project Research (0532-3) from Ibaraki Prefectural University of Health Sciences.

\section{References}

1. Struhl K: Histone acetylation and transcriptional regulation mechanisms. Genes Dev 12: 599-606, 1998.

2. Cress WD and Seto E: Histone deacetylases, transcriptional control, and cancer. J Cell Physiol 184: 1-16, 2000.

3. Marks P, Rifkind RA, Richon VM, Breslow B, Miller T and Kelly WK: Histone deacetylases and cancer: causes and therapies. Nat Rev Cancer 1: 194-202, 2001.

4. Xu WS, Parmigiani RB and Marks PA: Histone deacetylase inhibitors: molecular mechanisms of action. Oncogene 26 : 5541-5552, 2007.

5. Sugita K, Koizumi K and Yoshida H: Morphological reversion of sis-transformed NIH3T3 cells by trichostatin A. Cancer Res 52: 168-172, 1992.

6. Butler LM, Agus DB, Scher HI, Higgins B, Rose A, CordonCardo C, Thaler HT, Rifkind RA, Marks PA and Richon VM: Suberoylanilide hydroxamic acid, an inhibitor of histone deacetylase, suppresses the growth of prostate cancer cells in vitro and in vivo. Cancer Res 60: 5165-5170, 2000.

7. Burgess A, Ruefli A, Beamish H, Warrener R, Saunders N, Johnstone R and Gabrielli B: Histone deacetylase inhibitors specifically nonproliferating tumor cells. Oncogene 23: 6693-6701, 2004.

8. Zhang XD, Gillespie SK, Borrow JM and Hersey P: The histone deacetylase inhibitor suberic bishhydroxamate regulates the expression of multiple apoptotic mediators of melanoma cells Mol Cancer Ther 3: 425-435, 2004.

9. Xu W, Ngo L, Perez G, Dokmanovic M and Marks PA: Intrinsic apoptotic and thioredoxin pathways in human prostate cancer cell response to histone deacetylase inhibitor. Proc Natl Acad Sci USA 103: 15540-15545, 2006.

10. Donadelli M, Costanzo C, Beghelli S, Scupoli T, Dandrea M, Bonora A, Piacentini P, Budillon A, Caraglia M, Scarpa A and Palmieri M: Synergistic inhibition of pancreatic adenocarcinoma cell growth by trichostatin A and gemcitabine. Biochim Biophys Acta 1773: 1095-1106, 2007.

11. Zhang Y, Jung M and Dritschilo A: Enhancement of radiation sensitivity of human squamous carcinoma cells by histone deacetylase inhibitors. Radiat Res 161: 667-674, 2004.

12. Camphausen K, Burgan W, Cerra M, Oswald KA, Trepel JB, Lee MJ and Tofilon PJ: Enhanced radiation-induced cell killing and prolongation of $\gamma \mathrm{H} 2 \mathrm{AX}$ foci expression by the histone deacetyylase inhibitor MS-275. Cancer Res 64: 316-321, 2004.

13. Arundel GM, Glicksman AS and Leith JT: Enhancement of radiation injury in human colon tumor cells by the maturational agent sodium butyrate (NaB). Radiat Res 104: 443-448, 1985.
14. Munshi A, Kurland JF, Nishikawa T, Tanaka T, Hobbs ML, Tucker SL, Ismail S, Stevens C and Meyn RE: Histone deacetylase inhibitors radiosensitizes human melanoma cells by suppressing DNA repair activity. Clin Cancer Res 11: 4912-4922, 2005.

15. Munshi A, Tanaka T, Hobbs ML, Tucker SL, Richon VM and Meyn RE: Vorinostat, a histone deacetylase inhibitor, enhances the response of human tumor cells to ionizing radiation through prolongation of $\gamma$-H2AX foci. Mol Cancer Ther 5: 1967-1974, 2006.

16. Banuelos CA, Banáth JP, MacPhail SH, Zhao J, Reitsema T and Olive PL: Radiosensitization by the histone deacetylase inhibitor PCI-24781. Clin Cancer Res 13: 6816-6826, 2007.

17. Sun GH, Sohn TA, Ryu B and Kern SE: A nobel histone deacetylase inhibitor identified by high-throughput transcriptional screening of a compound library. Cancer Res 60: 3137-3142, 2000.

18. Keen JC, Yan L, Mack KM, Pettit C, Smith D, Sharma D and Davidson NE: A nobel histone deadetylase inhibitor, scriptaid, enhances expression of functional estrogen $\alpha(\mathrm{ER})$ in ER negative human breast cancer cells in combination with 5-aza-2'deoxycytidine. Breast Cancer Res Treat 81: 177-186, 2003.

19. Takai N, Ueda T, Nishida M, Nasu K and Narahara H: A nobel deacetylase inhibitor, scriptaid, induces growth inhibition, cell cycle arrest, and apoptosis in human endometric cancer and ovarian cancer cells. Int J Mol Med 17: 323-329, 2006.

20. Machida H, Matsumoto Y, Shirai M and Kubota N: Geldanamycin, an inhibitor of Hsp90, sensitizes human tumor cells to radiation. Int J Radiat Biol 79: 973-980, 2003.

21. Ungerstedt JS, Sowa Y, Xu WS, Shao Y, Dokmanovic M Perez G, Ngo L, Holmgren A, Jiang X and Marks PA: Role of thioredoxin in the response of normal and transformed cells to histone deacetylase inhibitors. Proc Natl Acad Sci USA 102: 673-678, 2005.

22. Girinsky T, Rubin L, Pignon JP, Chavaudra N, Gazeau J, Dubray B, Cosset JM, Socie G and Fertil B: Predictive value of in vitro radiosensitivity parameters in head and neck cancers and cervical carcinoma: preliminary correlations with local control and overall survival. Int J Radiat Oncol Biol Phys 25: 3-7, 1993.

23. Kim MS, Blake M, Baek JH, Kohlhagen G, Pommier Y and Carrier F: Inhibition of histone deacetylase increases cytotoxicity to anticancer drugs targeting DNA. Cancer Res 63: 7291-7300, 2003.

24. Dowdy SC, Jiang S, Zhou XC, Hou X, Jin F, Podratz KC and Jiang SW: Histone deacetylase inhibitors and paclitaxel cause synergistic effects on apoptosis and microtubule stabilization in papillary serous endometrial cancer cells. Mol Cancer Ther 5: 2767-2776, 2006.

25. Rogakou EP, Pilch DR, Orr AH, Ivanova VS and Bonner WM DNA double-stranded breraks induce histone H2AX phosphorylation on serine 139. J Biol Chem 273: 5858-5868, 1998.

26. Tuteja R and Tuteja $\mathrm{N}$ : Ku autoantigen: a multifunctional DNA binding protein. Crit Rev Biochem Mol Biol 35: 1-33, 2000.

27. Taccioli GE, Gottlieb TM, Blunt T, Priestley A, Demengeot, J, Mizuta R, Lehmann AR, Alt FW, Jackson SP and Jeggo PA: $\mathrm{Ku} 80$ : product of the XRCC5 gene and its role in DNA repair and V(D)J recombination. Science 265: 442-445, 1994.

28. Nussenzweig A, Sokol K, Burgman P, Li L and Li GC: Hypersensitivity of Ku80-deficient cell lien and mice to DNA damage: the effects of ionizing radiation on growth, survival, and development. Proc Natl Acad Sci USA 94: 13588-13590, 1997.

29. Wachsberger PR, Li WH, Guo M, Chen D, Cheong N, Ling CC, Li G and Iliakis G: Rejoining of DNA double strand breaks in Ku80-deficient mouse fibroblasts. Radiat Res 151: 398-497, 1999.

30. McKenna WG, Weiss MC, Endlich B, Ling CC, Bakanauskas VJ, Kelsten ML and Muschel RJ: Synergistic effect of the v-myc omcogene with H-ras on radioresistance. Cancer Res 50: 97-107, 1990.

31. Hermens AF and Bentvelzen PA: Influence of the H-ras oncogene on radiation response of a rat rhabdomyosarcoma cell line. Cancer Res 52: 3073-3082, 1992.

32. Chang IY, Youn CK, Kim HB, Kim MH, Cho HJ, Yoon Y, Lee YS, Chung MH and You HJ: Oncogenic H-ras up-regulates expression of Ku80 to protect cells from $\gamma$-ray irradiation in NIH3Ts cells. Cancer Res 65: 6811-6819, 2005. 